\title{
VALORACIÓN DE LA UTILIDAD DE LAS TUTORÍAS EN UNA EXPERIENCIA DE FORMACIÓN CON ESTUDIANTES DE 4 DE GRADO
}

Valuation of the utility of the tutories in a training experience with students of 4th grade

Avaliação da utilidade das tutorias numa experiência de formação com alunos do $4 .^{\circ}$ ano da Licenciatura

Laura Ruiz-Sanchis (1)

Concepción $\operatorname{Ros} \operatorname{Ros}(2)$

Julio Martín-Ruiz (3)

Apoyo/financiación del trabajo: Este estudio se ha llevado a cabo dentro del Convocatoria de Proyectos de Innovación Docente Universitaria -UCV: "Coordinación de materias de itinerario para un mejor desarrollo de las competencias profesionales del futuro Profesor de Educación Física". II Convocatoria Proyectos de Innovación Docente Universitaria y II Convocatoria de Premios a la Innovación Docente Universitaria, 2018-2019.

(1) Universidad Católica de Valencia San Vicente Mártir, España. Teléfono: +34 963637412. Correo electrónico: laura.ruiz@ucv.es

(2) Universidad Católica de Valencia San Vicente Mártir, España. Teléfono: +34 963637412. Correo electrónico: concepcion.ros@ucv.es

(3) Universidad Católica de Valencia San Vicente Mártir, España. Teléfono: +34 963637412. Correo electrónico: julio.martin@ucv.es

\section{Resumen}

Esta investigación analiza el nivel de utilidad que muestran las tutorías grupales dentro de la evaluación formativa de los estudiantes de $4^{\circ}$ de Grado Ciencias de Actividad Física y deporte (FCAFD), a partir del número de sesiones realizadas por las dos profesoras dentro del horario de las asignaturas como herramienta metodológica activa, y la importancia que le conceden para su desarrollo profesional, en función del número de tutorías a las que han acudido durante el diseño de una programación didáctica grupal. Se trata de una investigación descriptiva no experimental en el que se emplea una encuesta ad hoc con 15 ítems $( \pm=0,71)$. Las tutorías grupales son una herramienta efectiva para mejorar el rendimiento, reflexionar sobre el futuro académico y mejorar la relación con los compañeros.

Palabras clave: Educación Superior; tutorías grupales; evaluación continua

\begin{abstract}
The purpose of the study is the objective of knowing the level of usefulness shown by the group tutorials within the formative evaluation of the 4th Grade students Physical
\end{abstract}


Activity and Sports Sciences (FCAFD), based on the number of sessions held by the two teachers within the timetable of the subjects as an active methodological tool, and the importance they attach to their professional development, depending on the number of tutorials they have attended during the design of a group teaching program. This is a non-experimental descriptive research in which an ad hoc survey with 15 items $( \pm=$ 0.71. Group tutorials are an effective tool to improve performance, reflect on the academic future and improve the relationship with colleagues.

Keywords: Higher education; group tutorials; continuous assessment

\section{Resumo}

Esta investigação analisa o nível de utilidade demonstrado pelos tutoriais em grupo dentro da avaliação formativa dos alunos do $4 .^{\circ}$ ano da Licenciatura em Ciências da Atividade Física e Desporto (FCAFD), tendo por base o número de sessões realizadas por duas professoras, dentro do horário das disciplinas, como ferramenta metodológica ativa, e a importância que lhe atribuem para o seu desenvolvimento profissional, em função do número de tutoriais que participaram durante a elaboração de um programa de ensino em grupo. Trata-se de uma pesquisa descritiva não experimental, na qual se utiliza um questionário ad hoc com 15 itens $( \pm=0,71)$. As tutoriais em grupo são uma ferramenta eficaz para melhorar o desempenho, refletir sobre o futuro académico e melhorar a relação com os colegas.

Palavras-chave: Ensino superior; tutoriais em grupo; avaliação contínua

\section{Introducción}

La tutoría universitaria en sus distintas formas es una herramienta metodológica con un gran potencial (Álvarez, 2002; Pérez 2006) y se considera un factor de calidad en la innovación educativa (Aguaded \& Monescillo, 2013; Pino-Juste \& Soto-Carballo, 2010; Rodríguez-Espinar, 2008). La valoración por parte del alumnado de las tutorías universitarias, se evidencia en diferentes aspectos como el grado de satisfacción, sobre las aportaciones docentes, la utilidad de las tutorías (De la Torre-Laso, 2019); la empatía del tutor, el nivel de satisfacción de los alumnos, la disposición de los tutores hacia los alumnos (Benítez, 2018); la asesoría para la resolución de problemas de índole personal y académico, conocer el campo laboral (Pérez-Padilla, Tun-Colonia, BarreroSolís \& Salgado, 2017). 
El objetivo de este trabajo es analizar el nivel de utilidad de las tutorías grupales dentro de la evaluación formativa de los estudiantes de $4^{\circ}$ de Grado Ciencias de Actividad Física y deporte (FCAFD), en el itinerario de Enseñanza de la Actividad Física y el Deporte. El alumnado trabaja en las asignaturas de 'Pedagogía en Valores' y 'Diseño, evaluación e intervención de programas educativos' en grupos establecidos durante todo el cuatrimestre.

\section{Método}

La muestra son 36 estudiantes de la FCAFD, durante el curso 2018/2019. Se obtuvo un error muestral de 1,0\% para un nivel de confianza de 95\% $(p=0.05)$. Los estadísticos descriptivos generales indican que el 58,3\% de la muestra son hombres y el $41,7 \%$ son mujeres.

Las tutorías se realizan los martes y jueves, y se revisaban las prácticas de la semana anterior tras aplicar técnicas de aprendizaje cooperativo orientado a la resolución de problemas de la especialidad. Todos los miembros del grupo (4) debían participar en las tutorías, de entre 30 y 40 min. Finalizada la actividad se publicó, a través de un link, en la plataforma de la asignatura una encuesta de valoración de la utilidad de las tutorías grupales dentro de las sesiones realizadas. Se trata de una encuesta ad hoc con 15 ítems $( \pm=0,71)$, divididos en la información sociodemográfica (2) y la Utilidad de las tutorías (13). En ellas, se plantean preguntas con escala Likert del 1 al 5 (donde, 1 es baja, y 5 muy alta).

Se realizó un análisis descriptivo que recoge la Media $(M)$ y Desviación típica $(D T)$ de cada uno de los ítems a partir de la segregación de la muestra por el número de tutorías llevadas cabo con el grupo, prueba de Bonferroni, y análisis de Anova de un factor, para obtener información sobre la asistencia a la tutoría y la dimensión de la Utilidad. Para la realización de este proceso se aplicó el programa SPSS.v22.

\section{Resultados y discusión}

La distribución del número de tutorías a las que el alumnado asistió son: el $8 \%$ con una, el $24 \%$ con dos, el $34 \%$ con tres, el $19 \%$ con cuatro, el 15\% con más de cuatro. Dando la opción de acudir libremente, máximo una vez a la semana durante las 5 semanas de diseño del proyecto grupal. 
En la Tabla 1 se observan los datos descriptivos en relación al número de tutorías realizadas, con un rango de respuesta del 1 al 5:

Tabla 1.

Estadísticos descriptivos de Media y Desviación típica en relación a las tutorías realizadas

\begin{tabular}{|c|c|c|c|c|c|c|c|c|c|c|}
\hline \multirow[t]{2}{*}{ Ítem } & \multicolumn{2}{|c|}{1 tutoría } & \multicolumn{2}{|c|}{2 tutorías } & \multicolumn{2}{|c|}{3 tutorías } & \multicolumn{2}{|c|}{4 tutorías } & \multicolumn{2}{|c|}{$\begin{array}{l}\text { Más de } \\
\text { cuatro }\end{array}$} \\
\hline & $M$ & $D T$ & $M$ & $D T$ & $M$ & $D T$ & $M$ & $D T$ & $M$ & $D T$ \\
\hline item1_Mejora_rendimiento & 3,65 & 1,155 & 4,12 & .542 & 4,46 & .721 & 5,00 & .709 & 5,00 & .503 \\
\hline item2_Esfuerzo_resultado & 3,23 & .577 & 4,00 & .380 & 4,30 & .659 & 4,50 & .781 & 4,97 & .577 \\
\hline item3_Horarios_clase & 3,30 & .677 & 4,00 & .024 & 4,62 & .717 & 5,00 & .655 & 5,00 & 1,893 \\
\hline item4_Conocerme_mejor & 3,00 & .503 & 3,45 & .876 & 4,77 & .761 & 4,92 & .187 & 5,00 & .500 \\
\hline item5_Trabajo_grupal & 3,00 & .626 & 3,77 & .903 & 4,44 & .690 & 4,50 & .480 & 5,00 & .577 \\
\hline item6_Solución_dificultades & 3,88 & .535 & 4,00 & .779 & 4,98 & .576 & 5,00 & .120 & 5,00 & .335 \\
\hline item7_Conocer_normativa & 3,77 & .995 & 3,93 & .253 & 4,39 & .690 & 4,50 & .755 & 5,00 & .600 \\
\hline item8_Clase_presencial & 3,30 & .190 & 3,98 & .559 & 4,78 & .584 & 5,00 & .711 & 4,89 & 1,000 \\
\hline item9_Futuro_académico & 3,90 & .577 & 4,00 & .153 & 4,86 & .932 & 4,87 & .107 & 4,76 & .500 \\
\hline item10_Relación_compañeros & 3,20 & .692 & 3,73 & .294 & 4,34 & .550 & 4,50 & .548 & 4,99 & .760 \\
\hline item11_Cooperar_grupo & 3,23 & .575 & 3,80 & .221 & 4,37 & .637 & 4,50 & .678 & 4,78 & .577 \\
\hline $\begin{array}{l}\text { item12_Acompañado_profesor } \\
\text { as }\end{array}$ & 3,86 & .579 & 3,57 & .523 & 4,54 & .588 & 5,00 & .907 & 5,00 & .587 \\
\hline $\begin{array}{l}\text { item13_Comprendido_profesor } \\
\text { as }\end{array}$ & 3,53 & .577 & 4,00 & .473 & 4,50 & .659 & 5,00 & .711 & 5,00 & .500 \\
\hline
\end{tabular}

Nota: Media (M); Desviación típica (DT). El rango de respuesta del 1 al 5

En el grupo de alumnos que realizó una tutoría, el ítem más valorado fue el 9 "Reflexionar sobre mi futuro académico y profesional" $(M=3,90 ; D T=.577)$. Para el grupo con dos tutorías, el ítem con mayor puntuación fue el 1 "Mejorar mi rendimiento académico" $(M=4,12 ; D T=.542)$. Para el alumnado que acudió a tres tutorías, la valoración más alta fue el ítem 6 "Solucionar posibles dificultades con algunas prácticas del diseño de la Programación didáctica" $(M=4,98 ; D T=.576)$. Los valores más altos en el grupo con cuatro tutorías se encuentran en 6 ítems $(1,3,6,8,12$ y 13) con la máxima puntuación $(M=5,00)$, y finalmente, el alumnado con cuatro o más tutorías alcanza la máxima puntuación en 8 de los 13 ítems (1, 3, 4, 5, 6, 7, 12 y 13).

En la Figura 1 se aprecian las diferencias de valoración entre los 5 grupos participantes, indicando la puntuación Media de cada ítem.

Para conocer las valoraciones intergrupales entre las tendencias Utilidad y número de tutorías, se aplicó una Anova de un factor con la que se obtuvieron diferencias significativas en los ítems; $1(F=1140: p<.00), 9(F=2610: p<.01)$, y $10(F=2610$ : $p<.00$ ), para la puntuación media de alumnos que realizaron 4 sesiones frente a los que 
realizaron 1 tutoría grupal. Los resultados indican que el grupo de cuatro sesiones considera más útil las tutorías grupales que el de una. Sin embargo, a partir de la $4^{\mathrm{a}}$ sesión ya no se encuentran diferencias significativas.



Figura 1. Valores obtenidos en los ítems por cada grupo de participación en las tutorías.

\section{Conclusiones}

El alumnado considera que el modelo de tutoría grupal dentro del proceso de evaluación formativa contribuye positivamente a su mejora del rendimiento académico, a la reflexión sobre el futuro profesional y a las relaciones personales entre los miembros de un mismo grupo, pero también con el docente al sentirse más acompañados $\mathrm{y}$ comprendidos. Los procesos educativos que son continuos y se desarrollan de manera paralela benefician al alumnado, pues sienten que solucionan dificultades con algunas prácticas, mucho más que si son esporádicas.

La tutoría grupal hace posible la aplicación de la evaluación formativa y esto tiene notables repercusiones pedagógicas para el alumnado en formación.

\section{Referencias}

Aguaded, M. C. \& Monescillo, M. (2013). Evaluación de la tutoría en la Universidad de Huelva desde la perspectiva del alumnado de psicopedagogía: propuestas de mejora. Tendencias pedagógicas, 21, 163-176.

Álvarez, P. (2002). La función tutorial en la universidad; una apuesta por la mejora de la calidad de la enseñanza. Madrid: EOS. 
Benítez, S. M. (2018). Proceso enseñanza aprendizaje, formación y tecnologías de la información y la comunicación. Rev. cient. estud. investig., 7(1), 6-23. doi: 10.26885/rcei.7.1.6

De la Torre-Laso, J. (2019). La retroalimentación evaluative o feedback para los trabajos en grupo como estrategia de acción tutorial en la Universidad. Revista Educación, 43(1), 1-12.doi: 10.15517/revedu.v43i1.30062. Recuperado de https://revistas.ucr.ac.cr/index.php/educacion/article/view/30062/36963

Pérez, A. (2006). Tutorías. En M. de Miguel (Coord.) Metodologías de enseñanza y aprendizaje para el desarrollo de las competencias (pp. 133-167). Madrid: Alianza Editorial.

Pérez-Padilla E.A., Tun-Colonia J.A., Barrero-Solís C.L. \& Salgado H. (2017). Fortalezas y debilidades de un Programa de Tutorías en el nivel de Educación Superior: Una visión estudiantil. Revista Electrónica sobre Tecnología, Educación y Sociedad, 4(7), 1-21.

Pino Juste, M. \& Soto Carballo, J. (2010). Ventajas e inconvenientes de la tutoría grupal como estrategia docente. Estudio de caso. Bordón, 62(1), 155-166.

Rodríguez-Espinar, S. (2008). Manual de Tutoría Universitaria (2 $2^{\mathrm{a}}$ ed.). Barcelona: Octaedro/ICE-UB. 